\title{
Monitoramento de FitoplânCton e Microcistina No RESERVATÓRIO DA UHE AMERICANA ${ }^{1}$
}

\author{
Monitoring Phytoplankton and Microcistyn at the Americana Reservoir
}

\author{
FERREIRA, R.A.R. ${ }^{2}$, CAVENAGHI, A.L. ${ }^{3}$, VELINI, E.D. ${ }^{4}$, CORREAA, M.R. ${ }^{5}$, NEGRISOLI, E. ${ }^{5}$, \\ BRAVIN, L.F.N. ${ }^{6}$, TRINDADE, M.L.B. ${ }^{7}$ e PADILHA, F.S. ${ }^{8}$
}

\begin{abstract}
RESUMO - Este trabalho foi realizado na UHE Americana, pertencente à Companhia Paulista de Força e Luz, e faz parte de um projeto de pesquisa e desenvolvimento realizado em conjunto com a Faculdade de Ciências Agronômicas - UNESP, de Botucatu. As amostragens de água foram realizadas nos meses de fevereiro, abril, junho e outubro de 2004. As características analisadas foram: temperatura da água, pH, oxigênio dissolvido, condutividade, nitrogênio total, nitrito, nitrato, amônia, fósforo total, fosfato, fosfato inorgânico, juntamente com análise qualitativa e quantitativa da comunidade fitoplanctônica e a toxicidade. O reservatório apresentou valores elevados de fósforo total, variando de 18 a $509 \mu \mathrm{g} \mathrm{L}^{-1}$; fosfato, de 4 a $463 \mu \mathrm{g} \mathrm{L}^{-1}$; nitrogênio total, de 0,99 a 17,25 $\mathrm{mg} \mathrm{L}^{-1}$; e nitrato, de 0,26 a $15,29 \mathrm{mg} \mathrm{L}^{-1}$. Para a comunidade fitoplanctônica foram encontrados 103 táxons em todo o periodo amostrado; a maior riqueza foi encontrada no ponto P06, e a maior pobreza de táxons, nos pontos localizadas no corpo central do reservatório (P02, P03, P04 e P05). A maior concentração de cianoficea ocorreu em abril de 2004: 5.375.175 ind. $\mathrm{L}^{-1}$. As espécies que apresentaram as maiores densidades foram Microcystis aeruginosa, Anabaena spiroides, Microcystis sp. e Pseudoanabaena mucicola; a maior densidade foi apresentada por Anabaena spiroides, com 4.178.084 ind. $\mathrm{L}^{-1}$. Nos meses de junho e outubro a classe Cryptophyceae teve uma grande contribuição para a densidade total. Apesar da grande densidade de cianobactérias, os valores de toxicidade ficaram abaixo do limite permitido pela Portaria $n^{\circ} 1.469$.
\end{abstract}

Palavras-chave: fitoplâncton, microcistina, qualidade de água, reservatório.

\begin{abstract}
This work was carried out at the Americana Reservoir, owned by Companhia Paulista de Força e Luz, and was part of a joint R\&D project with Faculdade de Ciências Agronômicas Botucatu - São Paulo - Brazil. Water sampling was collected in February, April, June and October 2004. The following characteristics were analyzed: water temperature, $p H$, dissolved oxygen, conductivity, total nitrogen, nitrite, nitrate, ammonia, total phosphorus, inorganic phosphate, as well as qualitative and quantitative analysis of phytoplankton and toxicity. The reservoir showed high levels of total phosphorus, ranging from 18 to $509 \mu \mathrm{g} \mathrm{L}^{-1}$; phosphate, 4 to $463 \mu \mathrm{g} \mathrm{L}^{-1}$; total nitrogen, 0.99 to $17.25 \mathrm{mg} \mathrm{L}^{-1}$; nitrate, 0.26 to $15.29 \mathrm{mg} \mathrm{L}^{-1}$. For the phytoplankton community, 103 taxa were found during the sampled period, with the greatest richness being found at point $P 06$ and the poorest at the center of the reservoir (P02, P03, P04 and P05). The highest concentration of cyanophyceae was observed on April 2004, 5.375.175 un. $L^{-1}$. The species presenting higher densities were Microcystis aeruginosa, Anabaena spiroides, Microcystis sp. and Pseudoanabaena mucicola, with Anabaena spiroides presenting the highest density of all, 4.178.084 un. $L^{-1}$. In July and October, the Cryptophyceae class made a large contribution to total density. Despite the great density of Cyanobacteria, toxicity values were below the limit allowed by Ruling number 1.469.
\end{abstract}

Key words: phytoplankton, microcistyn, water quality, reservoir.

Recebido para publicação em 25.1.2005 e na forma revisada em 30.3.2005.

2 Pós-Graduação - CRHEA - Escola de Engenharia de São Carlos - USP <rosantonia@uol.com.br>; ${ }^{3}$ Eng.-Agr. Dr. - Dep. de Produção Vegetal - FCA/UNESP - Botucatu-SP; ${ }^{4}$ Professor Dr., Dep. de Produção Vegetal - FCA/UNESP - Botucatu-SP. ${ }^{5}$ PósGraduação - Dep. de Produção Vegetal - FCA/UNESP - Botucatu-SP; ${ }^{6}$ Professor M.S. - Faculdade de Ciências e Tecnologia de Botucatu-SP $;{ }^{7}$ Criativa - Consultoria em Matologia e Meio Ambiente; ${ }^{8}$ Companhia Paulista de Força e Luz.

Planta Daninha, Viçosa-MG, v. 23, n. 2, p. 203-214, 2005 


\section{INTRODUÇÃO}

A eutrofização do ecossistema aquático é o resultado do aumento da concentração de nutrientes, especialmente fósforo e nitrogênio. Esse processo de eutrofização interfere nas características físicas e químicas da água e, conseqüentemente, ocorrem profundas modificações qualitativas e quantitativas nas comunidades aquáticas.

Com o enriquecimento das águas superficiais (lagos, represas e rios) ocorre um crescimento não controlado de plantas aquáticas, com o florescimento de algas e cianobactérias que podem produzir toxinas nocivas ao homem e aos animais. Essas toxinas podem ser classificadas como hepatotoxinas, citotoxinas e endotoxinas e podem ser de difícil remoção, por serem estáveis e resistentes à hidrólise química ou oxidação (Tundisi, 2003). A microcistina já foi relatada como causadora de envenenamento de animais selvagens e domésticos; em fevereiro de 1996 foi constatada a morte de cerca de 50 pacientes hemodialisados devido à falência hepática, após receberem dose letal de microcistina intravenosa. Em 1999, a Organização Mundial de Saúde (OMS) estabeleceu o nível máximo aceitável de microcistina da água utilizada para o consumo humano como sendo de $1 \mu \mathrm{g} \mathrm{L^{-1 }}$ por dia, e a Portaria 1469/00 inseriu este limite nos procedimentos e responsabilidades relativos ao controle e à vigilância da qualidade da água para consumo humano (Magalhães et al., 2003).

O monitoramento das condições físicas, químicas e biológicas é importante, mas a classificação das algas, com suas flutuações no espaço e no tempo, é fundamental para a identificação das épocas favoráveis aos florescimentos e à concentração de toxinas na água (Tundisi, 2003).

A identificação e a quantificação das cianobactérias nos recursos hídricos são componentes principais para os programas de monitoramento e podem prever efetivamente o surgimento de florescimentos potencialmente tóxicos. Dados sobre as concentrações de fosfato total, nitrato e amônia são importantes para avaliar o potencial de desenvolvimento de cianobactérias e se espécies fixadoras ou não de nitrogênio podem aparecer (Lawton et al., 1999).
O objetivo deste trabalho foi realizar o monitoramento no reservatório da UHE Americana, em diferentes pontos, em quatro períodos de coleta, comparando a estrutura da comunidade fitoplanctônica em diferentes condições de trofia, por meio da densidade, diversidade, eqüidade e análise de toxicidade.

\section{MATERIAL E MÉTODOS}

O reservatório da UHE Americana está localizado na sub-bacia do rio Atibaia, no municipio de Americana (SP), a 22044'S e 47019'W. Possui uma bacia hidrográfica de aproximadamente $2,770 \mathrm{~km}^{2}$ e tem como principal afluente o rio Atibaia, que se apresenta num processo avançado de eutrofização, devido à intensa urbanização e industrialização em seu trecho inferior.

Na Figura 1 são apresentados os seis pontos selecionados para a coleta de amostras de fitoplâncton e microcistina, descritos como: P01: Jusante da Barragem - ponto com profundidade média de $0,5 \mathrm{~m}$ e presença de plantas marginais; P02: Barragem - ponto na Barragem, com profundidade de $15 \mathrm{~m}$ e presença de Salvinia sp.; P03: ponto com $7 \mathrm{~m}$ de profundidade média e presença de plantas marginais e flutuantes - água normalmente verde devido ao florescimento de cianobactérias; P04 e P05: profundidade média de $7 \mathrm{~m}$ e presença de plantas marginais e flutuantes; e P06: apresenta uma profundidade média de $1 \mathrm{~m}$, com grande quantidade de plantas aquáticas e de material em suspensão - ponto de entrada do reservatório que recebe água com alta carga de esgoto vindo do rio Atibaia.

A fim de avaliar as variações temporais e espaciais da comunidade fitoplanctônica, as variáveis fisicas e químicas e a toxicidade, as amostragens foram realizadas em fevereiro, abril, junho e outubro de 2004.

A transparência da água foi obtida através do disco de Secchi. O oxigênio dissolvido e a temperatura foram medidos com um oxímetro modelo Y-55 da YSI, e o pH e a condutividade, com medidores portáteis da marca Corning. Os nutrientes dissolvidos - nitrito, nitrato amônio, nitrogênio total, fosfato e fósforo total - foram determinados segundo Eaton et al. (1995). 


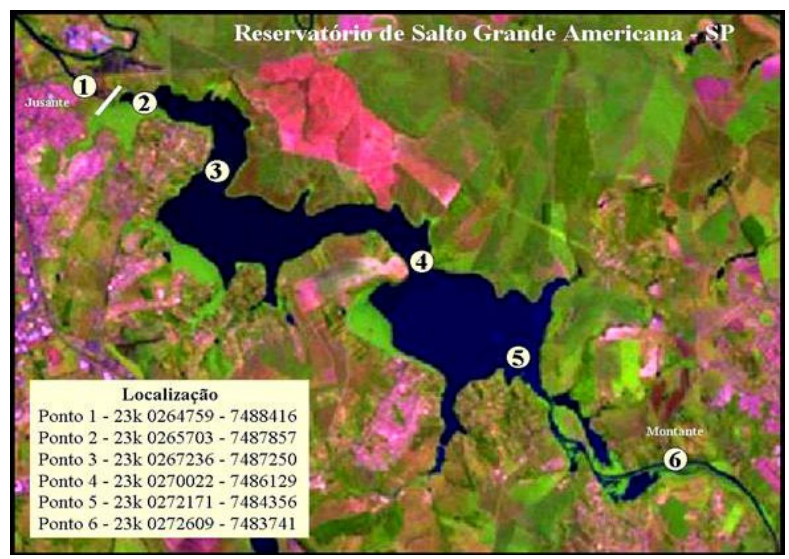

Figura 1 - Reservatório de Salto Grande, com a localização das estações de coleta.

Para a temperatura da água e oxigênio dissolvido, as medidas foram tomadas na superficie, a 2,5 e 5,0 $\mathrm{m}$ de profundidade; para as demais variáveis somente foi amostrada a superficie.

No caso de amostras qualitativas do fitoplâncton, foi utilizada a rede de $20 \mu \mathrm{m}$ de abertura da malha, e o material foi fixado com formol 4\% (Figura 2). A identificação foi feita em microscópio binocular (Zeiss) com aumento de até 1.000 vezes.

As amostras quantitativas foram coletadas na superficie e fixadas com lugol. A análise quantitativa foi realizada de acordo com o método descrito por Üthermohl (1958), que se baseia na distribuição aleatória dos indivíduos no fundo da câmara de sedimentação. A contagem foi realizada em microscópio invertido. $\mathrm{O}$ volume sedimentado variou de 2 a $40 \mathrm{~mL}$, dependendo da densidade de organismos ou detritos. O tempo de sedimentação foi no mínimo de três horas para cada centímetro de altura da câmara (Margalef, 1983).

Na contagem do fitoplâncton, consideraramse como um indivíduo organismos unicelulares, filamentos, tricomas, colônias e cenóbios.

Os campos de contagem foram distribuídos em transectos verticais paralelos, cobrindo praticamente toda a área da câmara. O número de campos contados foi o necessário para alcançar 100 indivíduos da espécie mais freqüente.

Para análise de microcistina, as amostras foram coletadas in natura (Figura 3), congeladas e enviadas para o laboratório responsável contratado para realização das análises.
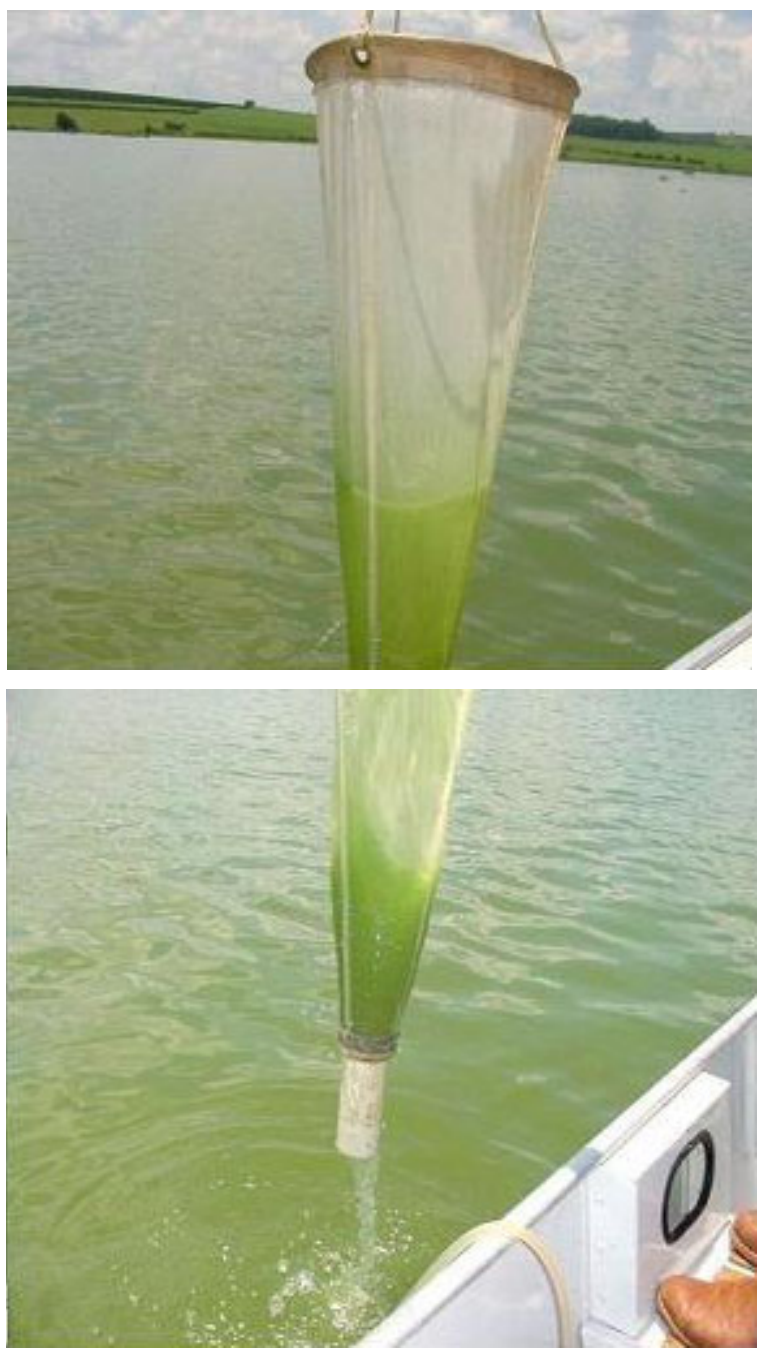

Figura 2 - Coleta da amostra de fitoplâncton - UHE Americana.

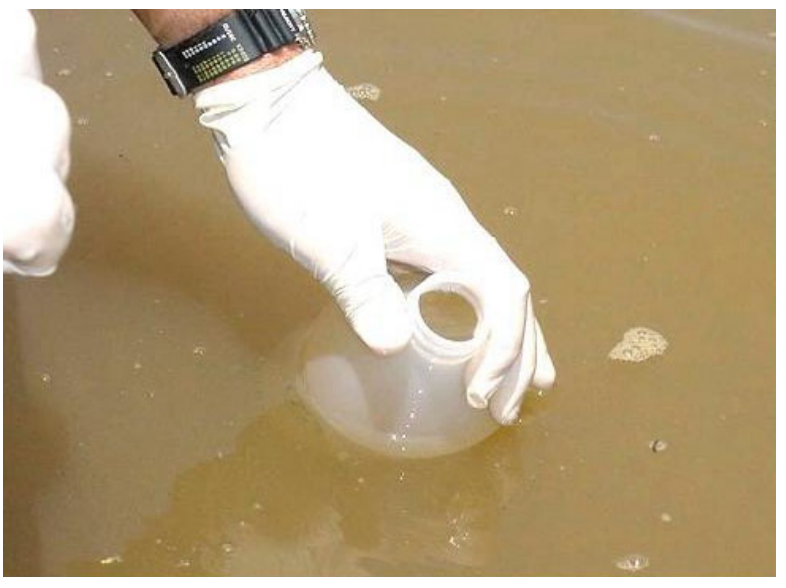

Figura 3 - Coleta da amostra para análise de microcistina UHE Americana.

Planta Daninha, Viçosa-MG, v. 23, n. 2, p. 203-214, 2005 


\section{RESULTADOS E DISCUSSÃO}

Na Tabela 1 são apresentados os dados de temperatura $\left({ }^{\circ} \mathrm{C}\right)$ e oxigênio dissolvido $\left(\mathrm{mg} \mathrm{L}^{-1}\right)$. Nos pontos P01 e P06 somente a superficie foi amostrada, devido à baixa profundidade deles. Para os pontos P02, P03, P04 e P05 a temperatura teve seus valores mais elevados em fevereiro, com uma tendência à formação de termoclina em fevereiro e abril de 2004. A maior temperatura ocorreu no ponto P05 $\left(28,4^{\circ} \mathrm{C}\right)$ a $2,5 \mathrm{~m}$ de profundidade no mês de fevereiro de 2004 , e a menor $\left(14,3{ }^{\circ} \mathrm{C}\right)$, na superficie do ponto P05 no mês de junho. O oxigênio dissolvido também apresentou as maiores concentrações em fevereiro no ponto P04, 7,41 $\mathrm{mg} \mathrm{L}^{-1}$ (superficie), e as menores concentrações também ocorreram em fevereiro, porém no ponto P02: $1,35 \mathrm{mg} \mathrm{L}^{-1}$ a $2,5 \mathrm{~m}$ de profundidade. O ponto P02 está localizado na região da barragem e, num estudo realizado anteriormente, Caligiuri et al. (1999) encontraram que este ponto apresentou anoxia a partir $9 \mathrm{~m}$ de profundidade tanto no inverno como no verão de 1996. Neste estudo, as concentrações de oxigênio dissolvido na barragem apresentaram oxiclina em todo o período amostrado.

Os valores de $\mathrm{pH}$ (Figura 4) foram maiores nos pontos P03, P04, P05 e P06 no mês de fevereiro, sendo o maior valor $(10,24)$ no ponto $\mathrm{P04}$, e os menores foram encontrados em junho, exceto o menor valor, que foi observado no ponto P06 em abril: 6,74. A condutividade $\left(\mathrm{mS} \mathrm{cm}^{-1}\right)$ está representada na Figura 5, onde se verifica que os maiores valores foram encontrados em outubro, sendo o ponto P06 aquele que apresentou sempre os maiores valores em todas as amostragens.

O nitrato e o nitrogênio total (Figura 6) tiveram maiores concentrações em fevereiro, com os maiores valores nos pontos P05 e P06. Para o nitrato a maior concentração foi de $15,34 \mathrm{mg} \mathrm{L}^{-1}$ no ponto P05 em fevereiro, e a menor, de $0,26 \mathrm{mg} \mathrm{L}^{-1}$ em outubro no ponto P01. $\mathrm{O}$ nitrogênio total foi maior no ponto P06 em fevereiro $\left(17,25 \mathrm{mg} \mathrm{L}^{-1}\right)$ e no ponto P02 em outubro $\left(0,99 \mathrm{mg} \mathrm{L}^{-1}\right)$. A maior concentração do nitrito foi encontrada no ponto P04 no mês de junho: $0,10 \mathrm{mg} \mathrm{L}^{-1}$. A amônia apresentou as maiores concentrações nos pontos mais a

Tabela 1 - Temperatura da água $\left({ }^{\circ} \mathrm{C}\right)$ e oxigênio dissolvido $\left(\mathrm{mg} \mathrm{L}^{-1}\right)$ encontrados nas três profundidade dos pontos de coleta durante o período amostrado

\begin{tabular}{|c|c|c|c|c|c|c|c|c|c|c|c|c|}
\hline \multirow{2}{*}{ Fev. de 2004} & \multicolumn{2}{|r|}{ P01 } & \multicolumn{2}{|r|}{$\mathrm{P} 02$} & \multicolumn{2}{|r|}{$\mathrm{P} 03$} & \multicolumn{2}{|r|}{ P04 } & \multicolumn{2}{|r|}{ P05 } & \multicolumn{2}{|r|}{ P06 } \\
\hline & $\mathrm{T}(\mathrm{C})$ & $\mathrm{O}_{2}\left(\mathrm{mg} \mathrm{L}^{-1}\right)$ & $\mathrm{T}(\mathrm{C})$ & $\mathrm{O}_{2}\left(\mathrm{mg} \mathrm{L}^{-1}\right)$ & $\mathrm{T}(\mathrm{C})$ & $\mathrm{O}_{2}\left(\mathrm{mg} \mathrm{L}^{-1}\right)$ & $\mathrm{T}(\mathrm{C})$ & $\mathrm{O}_{2}\left(\mathrm{mg} \mathrm{L}^{-1}\right)$ & $\mathrm{T}(\mathrm{C})$ & $\mathrm{O}_{2}\left(\mathrm{mg} \mathrm{L}^{-1}\right)$ & $\mathrm{T}(\mathrm{C})$ & $\mathrm{O}_{2}\left(\mathrm{mg} \mathrm{L}^{-1}\right)$ \\
\hline superfície & 21,8 & 4,10 & 22,1 & 5,40 & 24,7 & 7,10 & 25,7 & 7,41 & 27,1 & 6,75 & 24,7 & 3,13 \\
\hline 2,5 & & & 21,0 & 1,35 & 22,8 & 5,14 & 24,5 & 4,66 & 28,4 & 3,09 & & \\
\hline 5,0 & & & 20,9 & 1,52 & 23,9 & 3,85 & 24,6 & 2,25 & 23,6 & 2,65 & & \\
\hline \multirow{2}{*}{ Abr. de 2004} & \multicolumn{2}{|r|}{ P01 } & \multicolumn{2}{|r|}{$\mathrm{P} 02$} & \multicolumn{2}{|r|}{$\mathrm{P} 03$} & \multicolumn{2}{|r|}{ P04 } & \multicolumn{2}{|r|}{ P05 } & \multicolumn{2}{|r|}{ P06 } \\
\hline & $\mathrm{T}(\mathrm{C})$ & $\mathrm{O}_{2}\left(\mathrm{mg} \mathrm{L}^{-1}\right)$ & $\mathrm{T}(\mathrm{C})$ & $\mathrm{O}_{2}\left(\mathrm{mg} \mathrm{L}^{-1}\right)$ & $\mathrm{T}(\mathrm{C})$ & $\mathrm{O}_{2}\left(\mathrm{mg} \mathrm{L}^{-1}\right)$ & $\mathrm{T}(\mathrm{C})$ & $\mathrm{O}_{2}\left(\mathrm{mg} \mathrm{L}^{-1}\right)$ & $\mathrm{T}(\mathrm{C})$ & $\mathrm{O}_{2}\left(\mathrm{mg} \mathrm{L}^{-1}\right)$ & $\mathrm{T}(\mathrm{C})$ & $\mathrm{O}_{2}\left(\mathrm{mg} \mathrm{L}^{-1}\right)$ \\
\hline superfície & 22,2 & 4,33 & 22,1 & 4,28 & 23,2 & 5,91 & 24,2 & 6,21 & 25,4 & 5,88 & 25,4 & 3,55 \\
\hline 2,5 & & & 21,0 & 3,18 & 21,2 & 5,22 & 22,4 & 4,56 & 23,8 & 4,12 & & \\
\hline 5,0 & & & 20,9 & 2,32 & 19,2 & 4,71 & 21,0 & 4,23 & 22,4 & 4,36 & & \\
\hline \multirow{2}{*}{ Jun. de 2004} & \multicolumn{2}{|r|}{ P01 } & \multicolumn{2}{|r|}{$\mathrm{P} 02$} & \multicolumn{2}{|r|}{ P03 } & \multicolumn{2}{|r|}{ P04 } & \multicolumn{2}{|r|}{$\mathrm{P} 05$} & \multicolumn{2}{|r|}{ P06 } \\
\hline & $\mathrm{T}(\mathrm{C})$ & $\mathrm{O}_{2}\left(\mathrm{mg} \mathrm{L}^{-1}\right)$ & $\mathrm{T}(\mathrm{C})$ & $\mathrm{O}_{2}\left(\mathrm{mg} \mathrm{L}^{-1}\right)$ & $\mathrm{T}(\mathrm{C})$ & $\mathrm{O}_{2}\left(\mathrm{mg} \mathrm{L}^{-1}\right)$ & $\mathrm{T}(\mathrm{C})$ & $\mathrm{O}_{2}\left(\mathrm{mg} \mathrm{L}^{-1}\right)$ & $\mathrm{T}(\mathrm{C})$ & $\mathrm{O}_{2}\left(\mathrm{mg} \mathrm{L}^{-1}\right)$ & $\mathrm{T}(\mathrm{C})$ & $\mathrm{O}_{2}\left(\mathrm{mg} \mathrm{L}^{-1}\right)$ \\
\hline superficie & 19,1 & 4,54 & 18,3 & 4,55 & 16,3 & 4,84 & 15,6 & 5,09 & 14,3 & 5,44 & 13,9 & 3,79 \\
\hline 2,5 & & & 16,8 & 3,40 & 16,2 & 3,04 & 15,1 & 2,39 & 14,8 & 3,33 & & \\
\hline 5,0 & & & 15,9 & 1,82 & 14,8 & 1,96 & 14,5 & 2,18 & 14,4 & 3,06 & & \\
\hline \multirow{2}{*}{ Out. de 2004} & \multicolumn{2}{|r|}{ P01 } & \multicolumn{2}{|r|}{ P02 } & \multicolumn{2}{|r|}{$\mathrm{P} 03$} & \multicolumn{2}{|r|}{ P04 } & \multicolumn{2}{|r|}{ P05 } & \multicolumn{2}{|r|}{ P06 } \\
\hline & $\mathrm{T}(\mathrm{C})$ & $\mathrm{O}_{2}\left(\mathrm{mg} \mathrm{L}^{-1}\right)$ & $\mathrm{T}(\mathrm{C})$ & $\mathrm{O}_{2}\left(\mathrm{mg} \mathrm{L}^{-1}\right)$ & $\mathrm{T}(\mathrm{C})$ & $\mathrm{O}_{2}\left(\mathrm{mg} \mathrm{L}^{-1}\right)$ & $\mathrm{T}(\mathrm{C})$ & $\mathrm{O}_{2}\left(\mathrm{mg} \mathrm{L}^{-1}\right)$ & $\mathrm{T}(\mathrm{C})$ & $\mathrm{O}_{2}\left(\mathrm{mg} \mathrm{L}^{-1}\right)$ & $\mathrm{T}(\mathrm{C})$ & $\mathrm{O}_{2}\left(\mathrm{mg} \mathrm{L}^{-1}\right)$ \\
\hline superficie & 23,1 & 3,75 & 18,6 & 2,47 & 17,9 & 4,15 & 17,3 & 3,94 & 17,0 & 4,04 & 16,8 & 3,63 \\
\hline 2,5 & & & 18,1 & 3,32 & 17,8 & 4,89 & 17,2 & 4,17 & 16,8 & 3,53 & & \\
\hline 5,0 & & & 17,8 & 2,26 & 17,2 & 4,99 & 17,6 & 3,92 & 16,8 & 4,18 & & \\
\hline
\end{tabular}


montante da barragem (P04, P05 e P06), sendo no mês de outubro obtida a maior concentração $\left(1,89 \mathrm{mg} \mathrm{L}^{-1}\right)$ no ponto P06.

Para as concentrações de fósforo total, fosfato e fosfato inorgânico (Figura 7), as maiores concentrações ocorreram nos pontos P01, P05 e P06. O P06 teve os maiores valores destes nutrientes, sendo para o P-Total de $509 \mu \mathrm{g} \mathrm{L} \mathrm{L}^{-1}$; para o fosfato, de $463 \mu \mathrm{g} \mathrm{L} \mathrm{L}^{-1}$; e para o fosfato inorgânico, de $151 \mu \mathrm{g} \mathrm{L} \mathrm{L}^{-1}$. Para o P-total a menor concentração foi observada no ponto P04 em abril: $18 \mu \mathrm{g} \mathrm{L} \mathrm{L}^{-1}$.

A comunidade fitoplanctônica, considerando todo o período amostrado e todos os pontos, apresentou 103 táxons, sendo estes distribuidos da seguinte forma: a classe Bacillariophyceae contribuiu com 25 táxons $(24,3 \%)$; a Chlorophyceae, com 39 (37,9\%); a Zygnemaphyceae, com 4 (3,9\%); a Cyanophyceae, com $16(15,5 \%) ;$ a Cryptophyceae, com 7 (6,8\%); a Euglenophyceae,

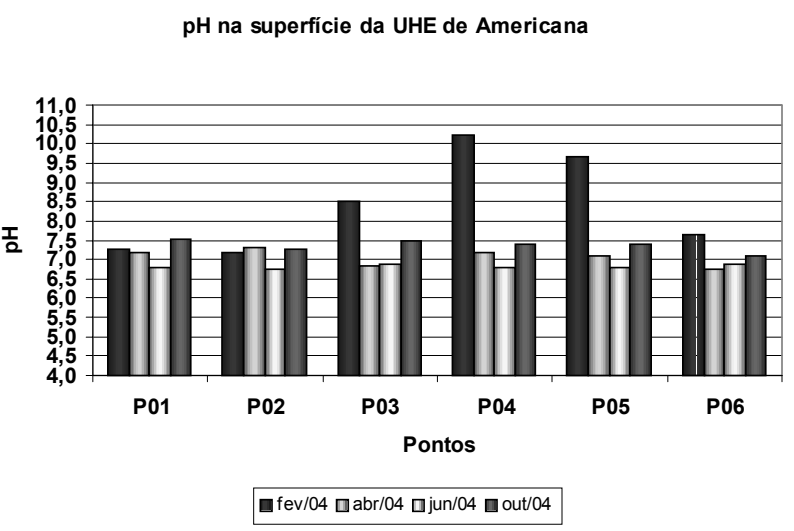

Figura 4 - Variação do pH dos pontos nos meses amostrados.

Condutividade $\left(\mathrm{mS} \mathrm{cm}^{-1}\right)$ na superfície da UHE de Americana

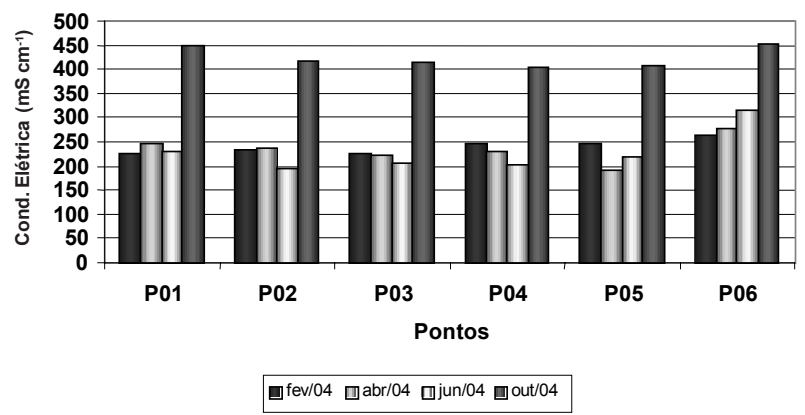

Figura 5 - Variação da condutividade elétrica $\left(\mathrm{mS} \mathrm{cm}^{-1}\right)$ dos pontos nos meses amostrados. com 9 (8,7\%); a Chrysophyceae, com $2(1,9 \%)$; e a Dinophyceae, somente com 1 (1\%). No geral, dos meses amostrados, fevereiro apresentou o maior número de táxons: 53.
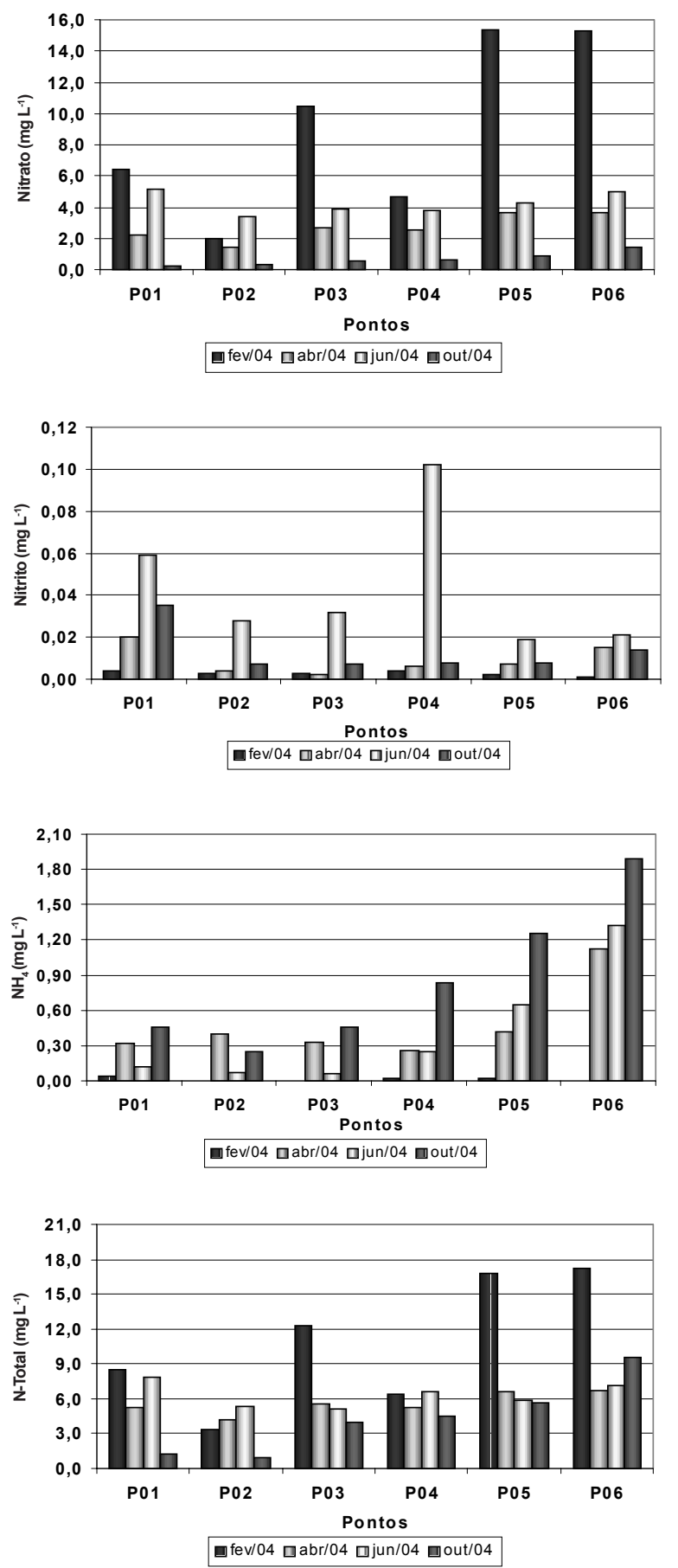

Figura 6 - Variações das concentrações de nitrato, nitrito, amônia e nitrogênio total $\left(\mathrm{mg} \mathrm{L}^{-1}\right)$ nos pontos, no período amostrado, na superfície da UHE de Americana.

Planta Daninha, Viçosa-MG, v. 23, n. 2, p. 203-214, 2005 


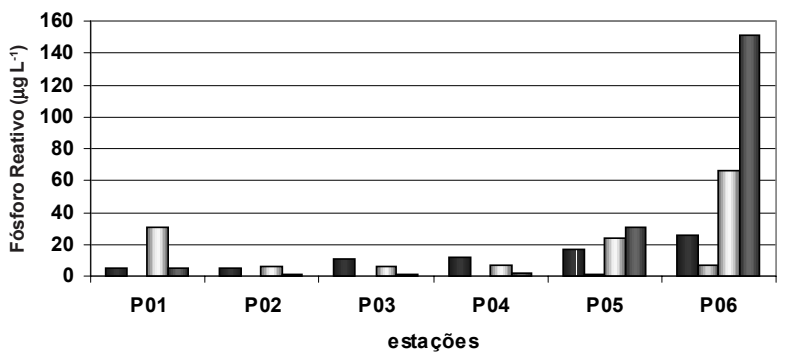

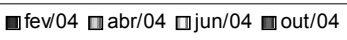

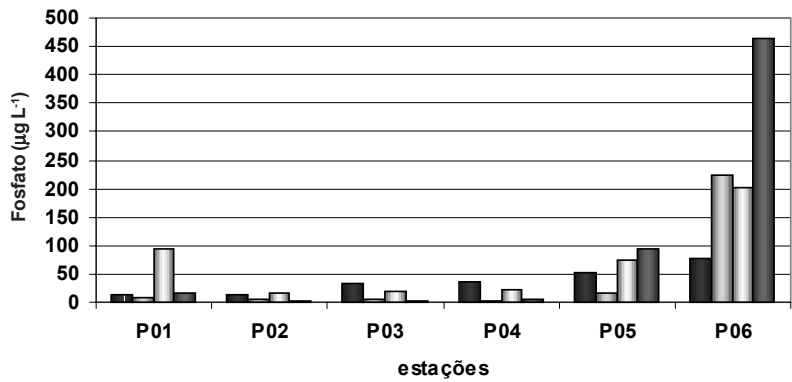

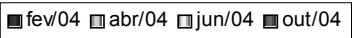

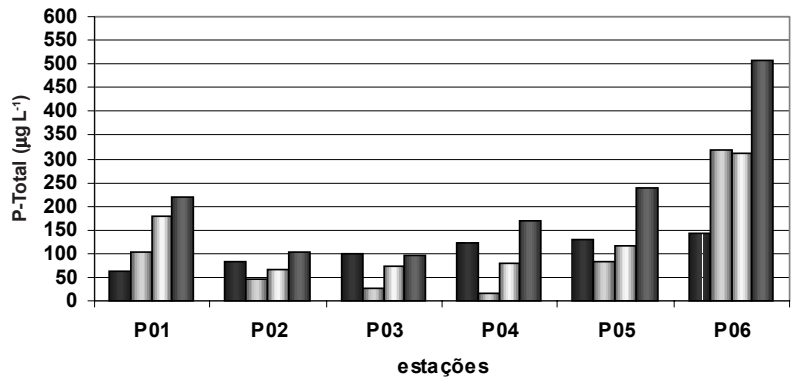

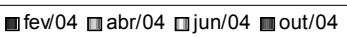

Figura 7 - Variações das concentrações do fosfato reativo, fosfato e fósforo total $\left(\mu \mathrm{g} \mathrm{L}^{-1}\right)$ nos pontos, no período amostrado, na superfície da UHE de Americana.

A variação da riqueza de espécies de fitoplâncton entre os pontos amostrados em cada mês (Figura 8) ocorreu da seguinte forma: as maiores riquezas foram encontradas primeiramente no ponto $\mathrm{P} 06$ e posteriormente no ponto P01. O maior número de táxons ocorreu em fevereiro no ponto P06 (36), e o menor, em fevereiro no ponto P03 (6).

A diversidade, de forma geral, também seguiu a mesma tendência da riqueza (Figura 9): o maior índice de diversidade foi obtido no mês de outubro no ponto P01 (4,09 bits por ind.) e o menor (1,91 bits por ind.) no ponto P03 em fevereiro. A eqüidade foi maior em outubro no ponto P01 $(0,904)$, e a menor $(0,528)$, no ponto P02 em abril.
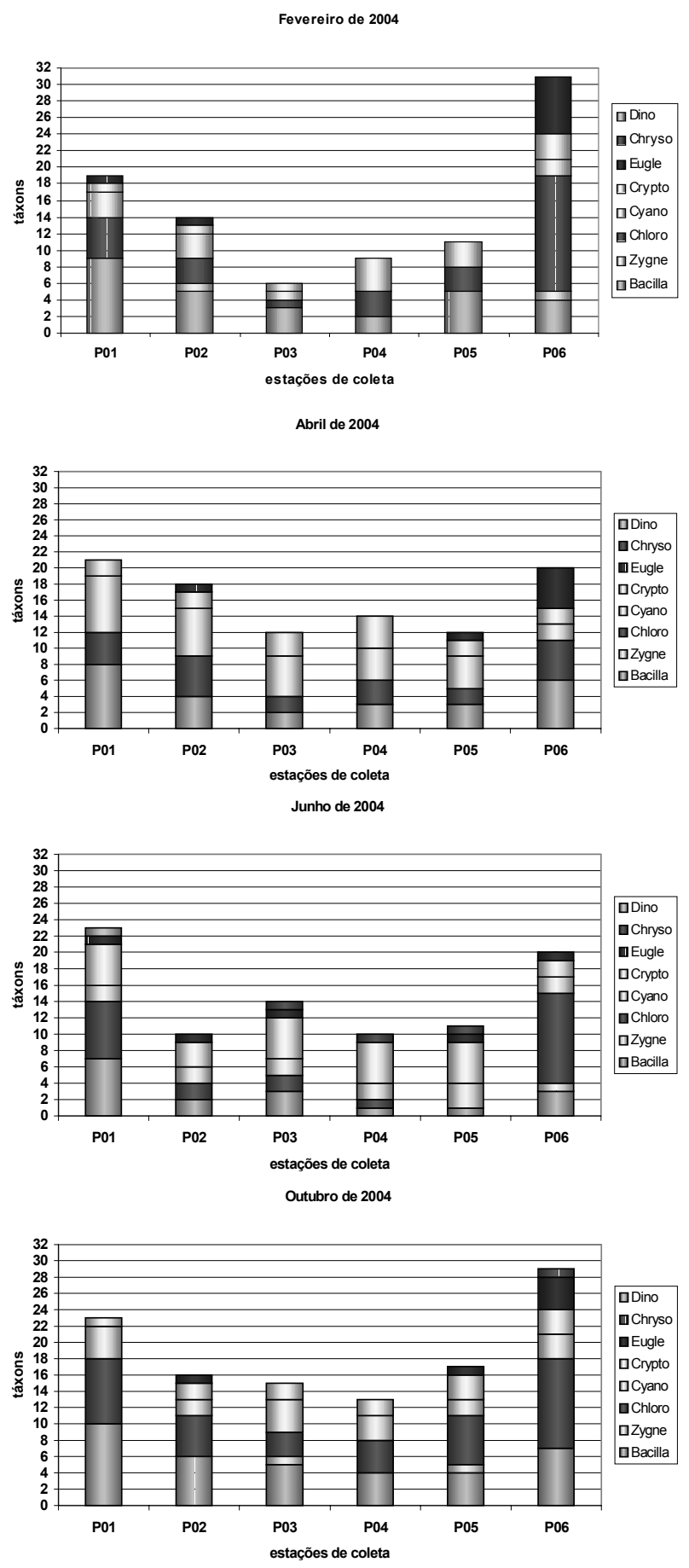

Figura 8 - Variação da riqueza de espécies nos pontos amostrados. 
Fevereiro de 2004

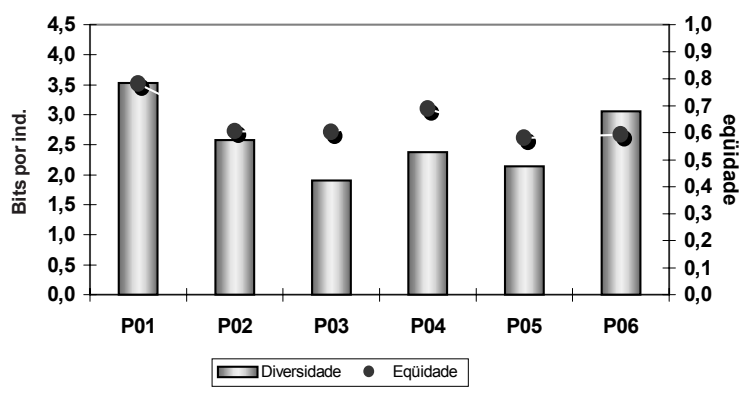

Abril de 2004

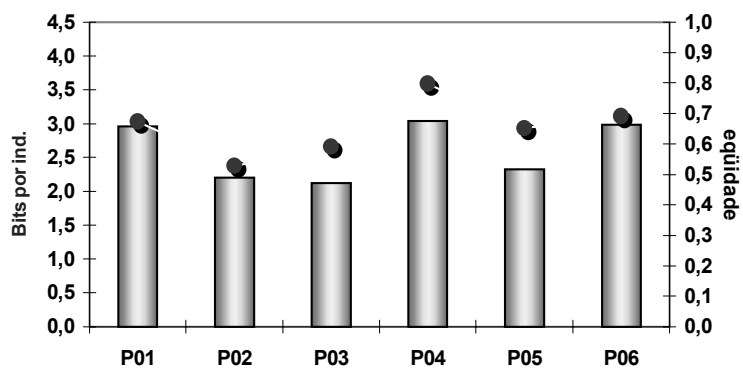

Junho de 2004

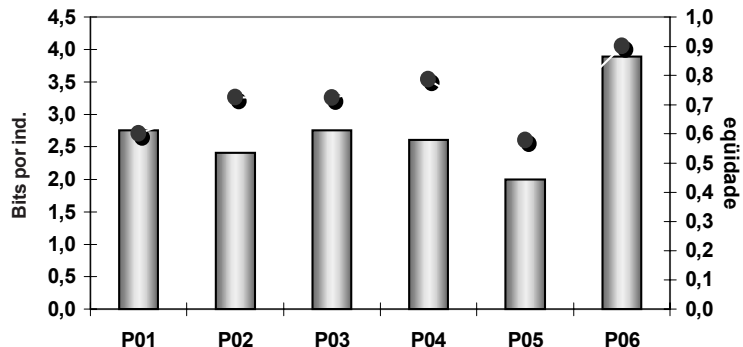

Outubro de 2004

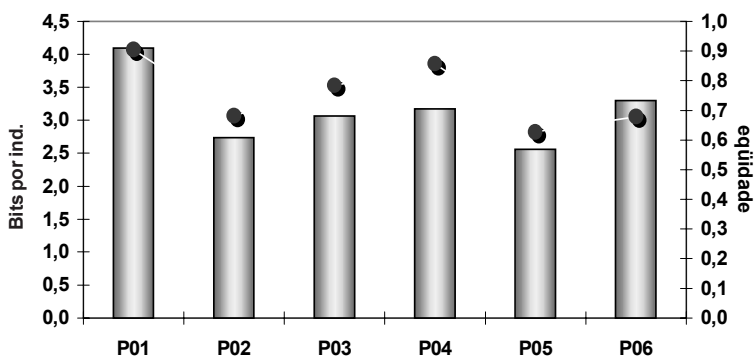

Figura 9 - Variação da diversidade e eqüidade nos pontos amostrados, na superfície da UHE de Americana.

Uma avaliação da baixa riqueza para os pontos situados mais ao centro do reservatório, somada às variáveis físicas e químicas destes, já indica que existe uma proliferação em grande densidade de algum grupo de algas (normalmente cianoficea).

Analisando os valores da densidade total do fitoplâncton, verificou-se que a partir do ponto P02 até o P05 houve dominância da classe Cyanophyceae nos meses de fevereiro e abril; nos meses de junho e outubro a classe Cryptophyceae teve aumento na sua densidade (exceto para o ponto P05 em junho e outubro). A maior densidade total foi encontrada em outubro no ponto P04: 8.555.214 ind. $\mathrm{L}^{-1}$; desta densidade total, 4.088 .889 ind. $\mathrm{L}^{-1}(47,8 \%)$ são da classe Cryptophyceae e 3.585.641 ind. $\mathrm{L}^{-1}$ da classe Cyanophyceae. A maior densidade de Cyanophyceae foi encontrada no ponto P04 (5.375.175 ind. $\mathrm{L}^{-1}$ ) em abril. A grande densidade de cianoficeas nos pontos P02, P03, P04 e P05 fez com que a riqueza de espécies fosse menor nesses pontos (Tabela 2 e Figuras 10 e 11).

As espécies de cianoficeas que tiveram as maiores densidades em fevereiro foram Microcystis aeruginosa e Anabaena spiroides; neste período, a maior densidade encontrada foi de Microcystis aeruginosa: 2.366.130 ind. $\mathrm{L}^{-1}$. Em abril, as espécies que tiveram as maiores densidade foram Anabaena spiroides, Microcystis aeruginosa e Pseudoanabaena mucicola, com a primeira apresentando a maior densidade: 4.178.084 ind. $\mathrm{L}^{-1}$, encontrada no P04. No mês de outubro, a maior densidade ocorreu no P05, onde Microcystis aeruginosa apresentou 1.864.713 ind. $\mathrm{L}^{-1}$, e Pseudoanabaena mucicola, 1.819.780 ind. $\mathrm{L}^{-1}$. No mês de outubro, as espécies com maiores densidade foram Microcystis aeruginosa, Anabaena spiroides e Oscillatoria cf. geminata; no P04, Microcystis aeruginosa teve densidade de 3.334.017 ind. $\mathrm{L}^{-1}$ (Tabelas 3, 4, 5 e 6).

De acordo com a Portaria n 1469 (BRASIL, 2000) que dispõe sobre procedimentos e responsabilidades inerentes ao controle e à vigilância da qualidade da água para consumo humano, o número de cianobactérias na água do manancial, no ponto de captação, não deve exceder 20.00 células $\mathrm{mL}^{-1}$.

Se for considerada somente a densidade de Microcystis aeruginosa no ponto P04 do mês de outubro, que foi de 3.334.017 ind. $\mathrm{L}^{-1}$, e transformá-la em células $\mathrm{mL}^{-1}$, o valor fica em 100.020 células $\mathrm{mL}^{-1}$, que é bem acima do permitido para a qualidade de água.

Planta Daninha, Viçosa-MG, v. 23, n. 2, p. 203-214, 2005 
Tabela 2 - Densidade das classes de fitoplâncton encontradas nos meses amostrados

\begin{tabular}{|c|c|c|c|c|c|c|}
\hline \multirow{2}{*}{ Classe } & \multicolumn{6}{|c|}{ Fitoplâncton (ind. $\mathrm{L}^{-1}$ ) } \\
\hline & $\mathrm{P} 01$ & $\mathrm{P} 02$ & $\mathrm{P} 03$ & P04 & P05 & P06 \\
\hline \multicolumn{7}{|c|}{ Fevereiro de 2004} \\
\hline Bacillariophyceae & 161.297 & 57.472 & 68.178 & 199.490 & 368.913 & 54.006 \\
\hline Zygnemaphyceae & 0 & 5.225 & 0 & 0 & 0 & 5.401 \\
\hline Chlorophyceae & 26.883 & 36.573 & 22.726 & 149.618 & 394.355 & 372.640 \\
\hline Cyanophyceae & 112.908 & 188.091 & 1.530 .227 & 3.723 .821 & 3.511 .032 & 10.801 \\
\hline Cryptophyceae & 10.753 & 15.674 & 7.575 & 0 & 0 & 847.891 \\
\hline Euglenophyceae & 5.377 & 5.225 & 0 & 0 & 0 & 205.222 \\
\hline Chrysophyceae & 0 & 0 & 0 & 0 & 0 & 0 \\
\hline Dinophyceae & 0 & 0 & 0 & 0 & 0 & 0 \\
\hline Total & 317.218 & 308.260 & 1.628 .707 & 4.072 .930 & 4.274 .299 & 1.495 .961 \\
\hline \multicolumn{7}{|c|}{ Abril de 2004} \\
\hline Bacillariophyceae & 200.593 & 58.517 & 93.610 & 375.558 & 498.006 & 92.652 \\
\hline Zygnemaphyceae & 0 & 0 & 0 & 0 & 0 & 0 \\
\hline Chlorophyceae & 57.312 & 37.238 & 18.722 & 70.417 & 52.422 & 34.135 \\
\hline Cyanophyceae & 447.036 & 638.369 & 767.603 & 5.375 .175 & 4.377 .208 & 9.753 \\
\hline Cryptophyceae & 11.462 & 85.116 & 74.888 & 610.282 & 707.692 & 156.046 \\
\hline Euglenophyceae & 0 & 5.320 & 0 & 0 & 26.211 & 48.764 \\
\hline Chrysophyceae & 0 & 0 & 0 & 0 & 0 & 0 \\
\hline Dinophyceae & 0 & 0 & 0 & 0 & 0 & 0 \\
\hline Total & 716.404 & 824.560 & 954.823 & 6.431 .433 & 5.661 .538 & 341.350 \\
\hline \multicolumn{7}{|c|}{ Junho de 2004} \\
\hline Bacillariophyceae & 317.383 & 34.948 & 39.316 & 49.403 & 909.890 & 26.687 \\
\hline Zygnemaphyceae & 0 & 0 & 0 & 0 & 0 & 3.812 \\
\hline Chlorophyceae & 67.756 & 20.969 & 15.122 & 13.723 & 0 & 87.687 \\
\hline Cyanophyceae & 7.132 & 160.760 & 120.973 & 112.528 & 3.779 .976 & 15.250 \\
\hline Cryptophyceae & 492.122 & 391.415 & 592.768 & 716.338 & 662.759 & 30.500 \\
\hline Euglenophyceae & 3.566 & 3.495 & 3.024 & 0 & 5.617 & 7.625 \\
\hline Chrysophyceae & 0 & 0 & 9.073 & 35.680 & 5.617 & 0 \\
\hline Dinophyceae & 3.566 & 0 & 0 & 0 & 0 & 0 \\
\hline Total & 891.525 & 611.586 & 780.276 & 927.671 & 5.363 .858 & 171.562 \\
\hline \multicolumn{7}{|c|}{ Outubro de 2004} \\
\hline Bacillariophyceae & 398.405 & 366.952 & 415.179 & 629.060 & 386.422 & 573.143 \\
\hline Zygnemaphyceae & 0 & 0 & 25.162 & 0 & 8.987 & 0 \\
\hline Chlorophyceae & 146.781 & 73.390 & 62.906 & 251.624 & 80.879 & 335.499 \\
\hline Cyanophyceae & 136.296 & 345.983 & 528.410 & 3.585 .641 & 799.805 & 55.916 \\
\hline Cryptophyceae & 73.390 & 828.262 & 1.446 .838 & 4.088 .889 & 242.637 & 754.872 \\
\hline Euglenophyceae & 0 & 10.484 & 0 & 0 & 8.987 & 139.791 \\
\hline Chrysophyceae & 0 & 0 & 0 & 0 & 0 & 41.937 \\
\hline Dinophyceae & 0 & 0 & 0 & 0 & 0 & 0 \\
\hline Total & 754.872 & 1.625 .071 & 2.478 .496 & 8.555 .214 & 1.527 .717 & 1.901 .159 \\
\hline
\end{tabular}


Fevereiro de 2004

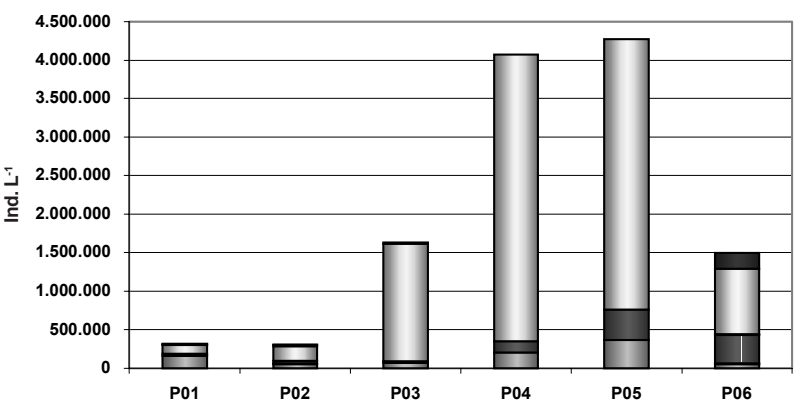

Abril de 2004

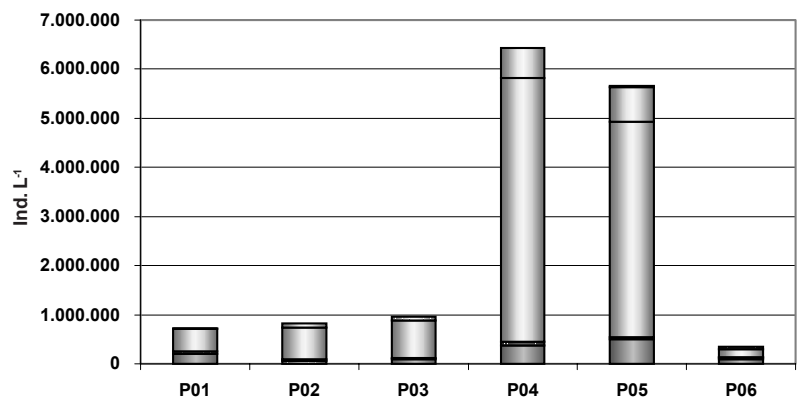

Junho de 2004

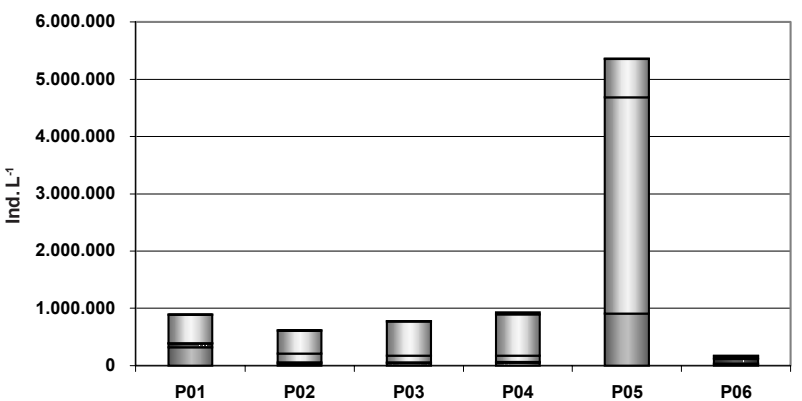

Outubro de 2004

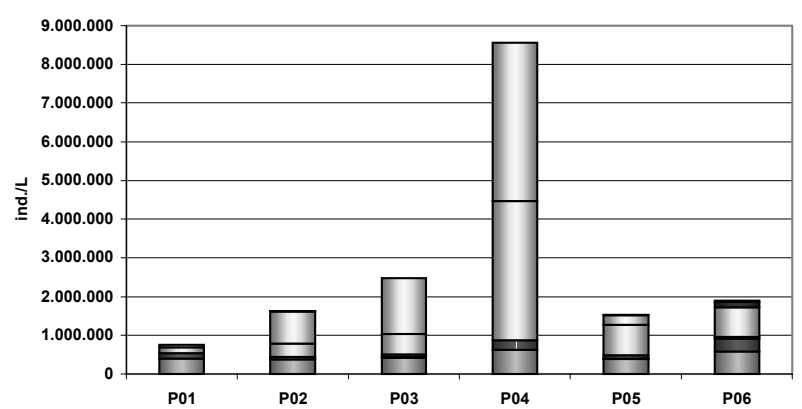

Figura 10 - Variação da densidade total do fitoplâncton (ind. $\mathrm{L}^{-1}$ ) nos pontos amostrados.

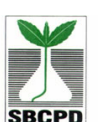

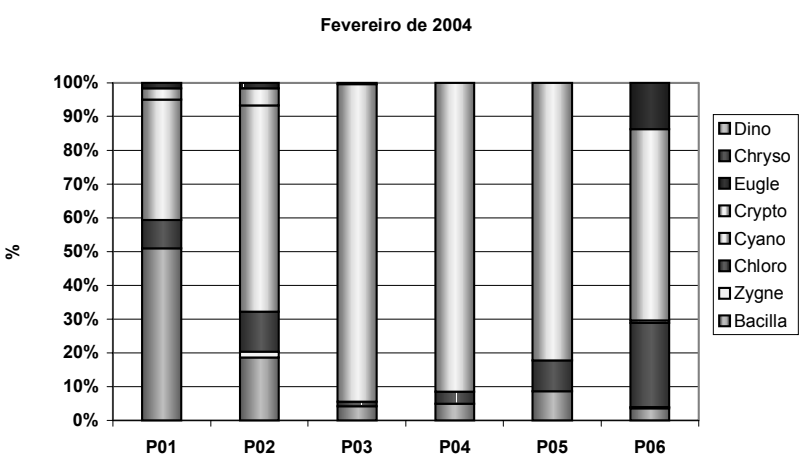
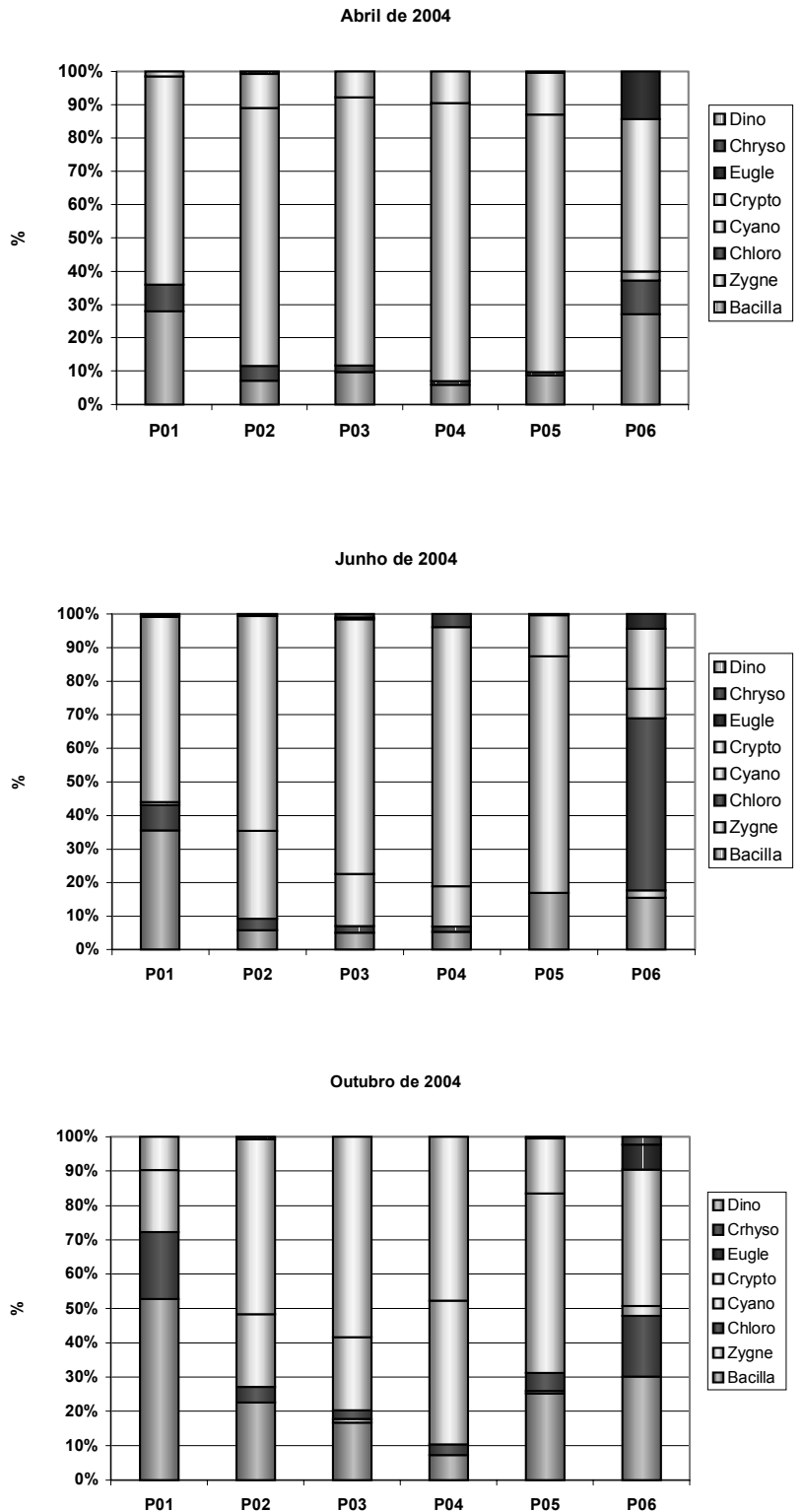

Figura 11 - Densidade relativa (\%) do fitoplâncton nos pontos amostrados.

Planta Daninha, Viçosa-MG, v. 23, n. 2, p. 203-214, 2005 
Tabela 3 - Densidade (ind. $\mathrm{L}^{-1}$ ) dos táxons da classe Cyanophyceae em fevereiro de 2004

\begin{tabular}{|c|c|c|c|c|c|c|}
\hline Cyanophyceae & $\mathrm{P} 01$ & $\mathrm{P} 02$ & $\mathrm{P} 03$ & P04 & $\mathrm{P} 05$ & P06 \\
\hline Anabaena spiroides & 91.402 & 167.192 & 1.530 .227 & 1.147 .070 & 1.043 .133 & \\
\hline Anabaena spp. & & & & & & 5.401 \\
\hline Aphanocapsa elachista & & & & & & \\
\hline \multicolumn{7}{|l|}{ Chroococcus spp. } \\
\hline \multicolumn{7}{|l|}{ Cylindrospermopsis raciborskii } \\
\hline Lyngbya largerheinii & & 10.449 & & & & \\
\hline \multicolumn{7}{|l|}{ Lyngbya spp. } \\
\hline Merismopedia tenuissima & 5.377 & & & & & \\
\hline Microcystis aeruginosa & & & & 2.177 .771 & 2.366 .130 & \\
\hline \multicolumn{7}{|l|}{ Microcystis incerta } \\
\hline Microcystis spp. & & & & 199.490 & & \\
\hline \multicolumn{7}{|l|}{ Oscillatoria cf. geminata } \\
\hline Oscillatoria spp. & 16.130 & 10.449 & & & & 5.401 \\
\hline Pseudoanabaena mucicola & & & & 199.490 & 101.769 & \\
\hline \multicolumn{7}{|l|}{ Pseudanabaena spp. } \\
\hline \multicolumn{7}{|l|}{ Raphidiopsis spp. } \\
\hline Total & 112.908 & 188.091 & 1.530 .227 & 3.723 .821 & 3.511 .032 & 10.801 \\
\hline
\end{tabular}

Tabela 4 - Densidade (ind. $\mathrm{L}^{-1}$ ) dos táxons da classe Cyanophyceae em abril de 2004

\begin{tabular}{|c|c|c|c|c|c|c|}
\hline Cyanophyceae & $\mathrm{P} 01$ & $\mathrm{P} 02$ & $\mathrm{P} 03$ & $\mathrm{P} 04$ & $\mathrm{P} 05$ & P06 \\
\hline Anabaena spiroides & 309.486 & 537.294 & 589.744 & 4.178 .084 & 1.100 .855 & \\
\hline \multicolumn{7}{|l|}{ Anabaena spp. } \\
\hline Aphanocapsa elachista & & 5.320 & & & & \\
\hline Chroococcus spp. & 40.119 & & & & & \\
\hline Cylindrospermopsis raciborskii & & & & & & 4.876 \\
\hline \multicolumn{7}{|l|}{ Lyngbya largerheinii } \\
\hline Lyngbya spp. & 5.731 & & & & & \\
\hline Merismopedia tenuissima & & & & & & 4.876 \\
\hline Microcystis aeruginosa & 28.656 & 47.878 & 28.083 & 633.754 & 1.258 .120 & \\
\hline \multicolumn{7}{|l|}{ Microcystis incerta } \\
\hline Microcystis spp. & 28.656 & 26.599 & (n) & 375.558 & 576.638 & \\
\hline \multicolumn{7}{|l|}{ Oscillatoria cf. geminata } \\
\hline Oscillatoria spp. & 11.462 & & & & & \\
\hline Pseudoanabaena mucicola & & 15.959 & 46.805 & 187.779 & 1.441 .595 & \\
\hline Pseudanabaena spp. & 22.925 & & 4.681 & & & \\
\hline Raphidiopsis spp. & & 5.320 & & & & \\
\hline Total & 447.036 & 638.369 & 767.603 & 5.375 .175 & 4.377 .208 & 9.753 \\
\hline
\end{tabular}

Tabela 5 - Densidade (ind. $\mathrm{L}^{-1}$ ) dos táxons da classe Cyanophyceae em junho de 2004

\begin{tabular}{|c|c|c|c|c|c|c|}
\hline Cyanophyceae & $\mathrm{P} 01$ & $\mathrm{P} 02$ & $\mathrm{P} 03$ & P04 & P05 & P06 \\
\hline \multicolumn{7}{|l|}{ Anabaena spiroides } \\
\hline \multicolumn{7}{|l|}{ Anabaena spp. } \\
\hline \multicolumn{7}{|l|}{ Aphanocapsa elachista } \\
\hline \multicolumn{7}{|l|}{ Chroococcus spp. } \\
\hline Cylindrospermopsis raciborskii & & & & & & 3.812 \\
\hline \multicolumn{7}{|l|}{ Lyngbya largerheinii } \\
\hline \multicolumn{7}{|l|}{ Lyngbya spp. } \\
\hline \multicolumn{7}{|l|}{ Merismopedia tenuissima } \\
\hline Microcystis aeruginosa & & 27.958 & 108.876 & 16.468 & 1.864 .713 & \\
\hline \multicolumn{7}{|l|}{ Microcystis incerta } \\
\hline Microcystis spp. & 3.566 & & & & 95.482 & \\
\hline \multicolumn{7}{|l|}{ Oscillatoria cf. geminata } \\
\hline Oscillatoria spp. & 3.566 & & & & & \\
\hline Pseudoanabaena mucicola & & 132.802 & 12.097 & 96.061 & 1.819 .780 & \\
\hline Pseudanabaena spp. & & & & & & 11.437 \\
\hline \multicolumn{7}{|l|}{ Raphidiopsis spp. } \\
\hline Total & 7.132 & 160.760 & $120 . .973$ & $112 . .528$ & 3.779 .976 & 15.250 \\
\hline
\end{tabular}

Planta Daninha, Viçosa-MG, v. 23, n. 2, p. 203-214, 2005 
Tabela 6 - Densidade (ind. $\mathrm{L}^{-1}$ ) dos táxons da classe Cyanophyceae em outubro de 2004

\begin{tabular}{|c|c|c|c|c|c|c|}
\hline Cyanophyceae & P01 & P02 & $\mathrm{P} 03$ & P04 & $\mathrm{P} 05$ & P06 \\
\hline Anabaena spiroides & 10.484 & & 100.650 & 62.906 & & \\
\hline \multicolumn{7}{|l|}{ Anabaena spp. } \\
\hline \multicolumn{7}{|l|}{ Aphanocapsa elachista } \\
\hline \multicolumn{7}{|l|}{ Chroococcus spp. } \\
\hline Cylindrospermopsis raciborskii & & & 12.581 & & & \\
\hline Lyngbya largerheinii & 31.453 & & & & & \\
\hline \multicolumn{7}{|l|}{ Lyngbya spp. } \\
\hline \multicolumn{7}{|l|}{ Merismopedia tenuissima } \\
\hline Microcystis aeruginosa & 62.906 & 325.014 & 339.692 & 3.334 .017 & 781.832 & 13.979 \\
\hline Microcystis incerta & & & & & 17.973 & \\
\hline \multicolumn{7}{|l|}{ Microcystis spp. } \\
\hline Oscillatoria cf. geminata & 31.453 & 20.969 & 75.487 & 188.718 & & 27.958 \\
\hline Oscillatoria spp. & & & & & & 13.979 \\
\hline \multicolumn{7}{|l|}{ Pseudoanabaena mucicola } \\
\hline \multicolumn{7}{|l|}{ Pseudanabaena spp. } \\
\hline \multicolumn{7}{|l|}{ Raphidiopsis spp. } \\
\hline Total & 136.296 & 345.983 & 528.410 & 3.585 .641 & 799.805 & 55.916 \\
\hline
\end{tabular}

Apesar de haver uma grande densidade de cianoficeas no reservatório da UHE Americana, os valores de toxicidade não foram significativos em todo o período, já que o permitindo por lei é de $1 \mu \mathrm{g} \mathrm{L}{ }^{-1}$ e o encontrado esteve sempre abaixo deste limite (Tabela 7 ).

Quando ocorre morte ou lise celular, há a liberação de toxinas, que podem lesar o fígado (hepatotoxinas), o sistema nervoso (neurotoxinas) ou somente irritar a pele. Apenas 30 a $50 \%$ das espécies de cianobactérias produzem essas toxinas, porém a única forma de saber se uma colônia de alga está produzindo toxina é através da análise laboratorial. A intoxicação por cianobactérias não é algo

Tabela 7 - Resultado da análise de microcistina $\left(\mu \mathrm{g} \mathrm{L}^{-1}\right)$ das amostras coletadas em superfície no reservatório de Salto Grande, Americana-SP, em 2004

\begin{tabular}{|c|c|c|c|c|}
\hline Ponto & Fevereiro & Abril & Junho & Outubro \\
\hline P01 & - & $* *<0,5$ & $* *<0,5$ & $* *<0,5$ \\
\hline P02 & $*<$ L.D. & $* *<0,5$ & $* *<0,5$ & $* *<0,5$ \\
\hline P03 & 0,176 & $* *<0,5$ & $* *<0,5$ & $* *<0,5$ \\
\hline P04 & 0,205 & $* *<0,5$ & $* *<0,5$ & $* *<0,5$ \\
\hline P05 & 0,179 & $* *<0,5$ & $* *<0,5$ & $* *<0,5$ \\
\hline P06 & $*<$ L.D. & $* *<0,5$ & $* *<0,5$ & $* *<0,5$ \\
\hline
\end{tabular}

* <L.D. = limite de detecção de $0,118 \mu \mathrm{g} \mathrm{L}^{-1}$ - Laboratório ATM Rio de Janeiro-RJ; e $* *<0,5 \mathrm{ppb}=$ limite de deteç̧ão de $0,5 \mu \mathrm{g} \mathrm{L}^{-1}-$ Laboratório Tema - Americana-SP. recente; há descrições que datam do século XII. Como esta alga prolifera mais facilmente em água rica de nutrientes (devido ao fosfato presente em detergentes ou fertilizantes), esse problema está longe de ser resolvido em nosso meio. Portanto, a presença de microcistina e fitoplâncton com potencial de produção desta toxina indica a necessidade de monitoramento delas no reservatório da UHE Americana.

\section{AGRADECIMENTOS}

À CPFL, Companhia Paulista de Força e Luz, pelo apoio técnico e financeiro. Projeto financiado com recursos da linha P \& D da ANEEL.

\section{LITERATURA CITADA}

BRASIL. Ministério da Saúde. Portaria nº $1.469-$

Controle e vigilância da qualidade da água para consumo humano e seu padrão de potabilidade. Dezembro de 2000. Disponível em:

$<$ www.sbn.org.br/Portarias/portaria34.htm>. Acesso em: 10 fev. 2004.

CALIJURI, M. C.; DEBERDT, G. L. B.; MINOTI, R. T. A produtividade primária na represa de Salto Grande. In: HENRY, R. (Ed.). Ecologia de reservatórios: estrutura, função e aspectos sociais. Botucatu: FAPESP/ FUNDIBIO, 1999. p. 109-148.

EATON, A. D.; CLESCERI, L. S.; GREENBERG, A. E. (Eds.). Standard methods for the examination of water and wastewater. 19.ed. Maryland: American Public Health Association, 1995. $106 \mathrm{p}$.

Planta Daninha, Viçosa-MG, v. 23, n. 2, p. 203-214, 2005 
LAWTON, L. et al. Determination of Cyanobacteria in the laboratory. In: CHORUS, I.; BARTRAM (Eds.) Toxic cyanobacteria in water. London: E \& Spon, New Fetter Lane, 1999. $416 \mathrm{p}$.

MAGALHÃES, V. F. et al. Avaliação da presença de microcistina no reservatório do Funil e na Estação de Tratamento de Água do Guandu. In: CONGRESSO BRASILEIRO DE LIMNOLOGIA, 9., 2003. Juiz de ForaMG. Anais... Juiz de Fora: SBL, 2003. CD-ROM
MARGALEF, R. Limnologia. Barcelona: Omega, 1983. $1.010 \mathrm{p}$.

TUNDISI, J. G. Água no século XXI: enfrentando a escassez. São Carlos: RiMa, IIE, 2003. 248 p.

UTHERMÖHL, H. Zur Vervollkomninung der quantitativen. Phytoplankton - Methodik. Mitt. Int. Ver. Limnol., v. 9, 1958. 38 p. 